\title{
À la recherche d'une iconographie « incroyable » et " merveilleuse »: les panneaux décoratifs sous le Directoire
}

Anne Lafont

\section{(2) OpenEdition \\ Journals}

Édition électronique

URL : https://journals.openedition.org/ahrf/2035

DOI : 10.4000/ahrf.2035

ISSN : 1952-403X

Éditeur :

Armand Colin, Société des études robespierristes

Édition imprimée

Date de publication : 1 juin 2005

Pagination : 5-21

ISSN : 0003-4436

\section{Référence électronique}

Anne Lafont, « À la recherche d'une iconographie « incroyable » et « merveilleuse » : les panneaux décoratifs sous le Directoire », Annales historiques de la Révolution française [En ligne], 340 | avril-juin 2005, mis en ligne le 15 juin 2008, consulté le 22 avril 2022. URL : http://journals.openedition.org/ahrf/ 2035 ; DOI : https://doi.org/10.4000/ahrf.2035

Ce document a été généré automatiquement le 22 avril 2022.

Tous droits réservés 


\title{
À la recherche d'une iconographie « incroyable » et " merveilleuse» : les panneaux décoratifs sous le Directoire
}

\author{
Anne Lafont
}

1 La plupart des travaux consacrés à l'art de la fin du XVIII ${ }^{e}$ siècle interrogent sa dimension publique : la réception des expositions au Salon sous la Révolution ${ }^{1}$, le déroulement des concours, les rapports entre l'art et le politique et l'activité militante des artistes ${ }^{2}$. Or, il est une production moins bien connue, celle des ensembles décoratifs destinés aux appartements privés de personnalités politiques et financières, qui ont émergé avec le Directoire, après s'être enrichies grâce au commerce de fournitures aux armées et à la spéculation sur les biens nationaux.

2 Ces «parvenus », conscients de l'aura d'un luxe ostentatoire, surent fort bien s'arroger le concours des artistes pour décorer leurs hôtels particuliers, qui devinrent de petites cours, des lieux d'influence, dont le décor ne fut pas laissé au hasard. Aussi, il paraitt important, avant d'en interpréter le fonctionnement symbolique, d'identifier les panneaux décoratifs, les metteurs en scène (clients et architectes) et les auteurs proprement dits, c'est-à-dire les peintres.

Nature des objets

3 Le panneau décoratif a toujours été conçu comme la partie d'un ensemble homogène. Par conséquent, le projet de tableau est soumis à une destination précise (telle cimaise) et à un thème global, en rapport avec la fonction de la pièce et décliné sous différentes formes : mobilier, tapis, tapisseries, objets décoratifs... Le panneau peut être réalisé par un artiste peintre (c'est-à-dire un auteur) ou par un peintre décorateur (c'est-à-dire un interprète exécutant des motifs imaginés par le « metteur en scène »). Cette répartition du travail a toujours prévalu et renseigne sur l'approche méthodologique à adopter quand on étudie cette production, dont la part la plus originale revient bien sûr aux 
tableaux d'auteurs, même si une compréhension fine de ces ensembles est impossible sans la connaissance de toutes les variantes peintes.

4 Pour la Révolution, on connaît les acteurs de ces décorations privées et on documente assez précisément leurs participations respectives : Charles Percier et Pierre Fontaine, célèbres architectes d'intérieur sous l'Empire, se firent connaître grâce à leur contribution à la transformation de l'Hôtel Bourbon en Assemblée nationale sous la direction des architectes Lecomte et Gisors, au début du Directoire. Les premiers clients de Percier et Fontaine furent les financiers Ouvrard, Chauvelin et Gaudin, qui firent aménager et décorer leurs hôtels récemment acquis dans le quartier de la Chaussée d'Antin $^{3}$. Ces financiers furent à l'origine de la fortune des deux artistes auprès du couple Bonaparte et de leur entourage ${ }^{4}$.

Opportunément, la classe des enrichis du Directoire, qui devait bientôt constituer l'élite consulaire, s'appropria le quartier de la Chaussée d'Antin, qui avait accueilli, au cours des trente dernières années de l'Ancien Régime, les financiers et les comédiennes d'alors. Jean-Joseph de Laborde, Madame de Montesson (maîtresse puis épouse secrète du duc d'Orléans), la Guimard, Necker, Madame de Thélusson, parmi d'autres, avaient occupé des «folies» édifiées par Brongniart, Ledoux ou Pérard de Montreuil, sur les rue de Provence et de la Chaussée d'Antin ${ }^{5}$. Ce quartier, en plein essor au moment de la prise de la Bastille, se caractérisait par la concentration d'un luxe arrogant et soudain, comme l'a fort bien montré Louis-Sébastien Mercier, l'auteur du Tableau de Paris ${ }^{6}$, contrairement au quartier de Saint-Germain-des-Prés, plus ancré dans une tradition aristocratique ${ }^{7}$. Aussi, il n'est pas surprenant que la rupture de 1789 ait seulement suspendu quelques années l'effervescence de ce quartier disponible à la surenchère luxueuse. La légitimation du pouvoir autocratique de Bonaparte passa toujours par la récupération des attributs monarchiques d'Ancien Régime : l'investissement clanique du quartier de Paris le plus prisé dans les années quatre-vingt en est un des premiers exemples ${ }^{8}$. Par conséquent, les lieux d'investissement de l'élite intellectuelle, artistique, financière et politique demeurèrent les mêmes, avant et après 1789, comme on observe une véritable constante iconographique, si ce n'est stylistique, dans les choix décoratifs de ces appartements.

6 Les artistes français de la fin du XVIII ${ }^{e}$ siècle puisèrent leurs modèles dans la tradition italienne renaissante (Botticelli, Raphaël, Titien), mais aussi dans les exemples fournis par la génération ayant précédé Jacques-Louis David, comme Joseph-Marie Vien, Louis Lagrenée, ou même, au strict plan thématique, François Boucher. Effectivement, les Heures du jour ou les Saisons, et les Muses avaient l'avantage d'être des personnifications féminines de valeurs ou de qualités récupérables en toute occasion. En outre, elles se présentaient sous forme de cycle de trois à neuf unités (trois ou quatre heures du jour, quatre saisons et jusqu'à neuf muses), si bien que la série pouvait être modulée en fonction du nombre de cimaises à parer, ce que les décorateurs de la Ferrare du XV siècle jusqu'à Pierre-Paul Prudhon dans l'hôtel Lannoy de la rue Cerrutti (actuelle rue Lafitte) comprirent fort bien ${ }^{9}$. D'ailleurs, la preuve la plus flagrante de continuité et de remplois décoratifs dans la France de la deuxième moitié du XVIII ${ }^{\mathrm{e}}$ siècle - malgré la Révolution - se révèle dans la fortune de la frise anonyme du château de Rueil-Malmaison (illustration 1). Cette peinture, inspirée du mode figuratif des Noces aldobrandines, représente les Arts, les Muses, Apollon, Vénus, Cupidon... ${ }^{10}$. Elle fut conçue vers 1780 pour décorer le salon privé de Julie Carreau (future épouse de Talma), du temps où elle vivait en compagnie d'Alexandre de Ségur, rue Chantereine. Le couple 
de comédiens cohabita ensuite dans cette même demeure et, à la suite de leur divorce, Julie, propriétaire de la maison construite par l'architecte Pérard de Montreuil, la loua à Joséphine et Napoléon Bonaparte, qui s'y installèrent au moment de leur mariage au début de l'année $1796{ }^{11}$. Malgré le renouvellement décoratif orchestré par Jacob et commandé par Joséphine depuis Milan, où elle accompagnait son mari pendant la campagne d'Italie, la frise resta intacte, à tel point qu'elle fut démantelée seulement sous le Second Empire (au moment de la destruction de la maison), puis finalement donnée au musée de Malmaison (en 1961), en souvenir de la première demeure des Bonaparte.

7 D'autres exemples prouvent que, dans le domaine de la décoration, il n'y eut pas de «Révolution du goût ». Si certaines transformations formelles sont perceptibles dans le volume des corps féminins ou dans l'opacité des couleurs - entre Vien et Meynier notamment - globalement, leurs choix stylistiques sont proches et leur écart n'est pas plus excessif que celui qui sépare, traditionnellement, des artistes de deux ou trois générations successives. Aussi, les panneaux que Vien conçut au début des années soixante-dix pour Madame du Barry ${ }^{12}$ préfigurent ceux récemment acquis par le Musée de Cleveland, que Meynier avait réalisés pour le salon privé de Boyer-Fonfrède, banquier à Toulouse ${ }^{13}$. Dans les deux cas, les personnifications féminines, aussi connotées soient-elles, conviennent à ces décors intérieurs où, avant et après 1789 , les motifs de femme se prêtent décidément bien à l'aspect ornemental des salons privés. Les neuf muses de Boyer-Fonfrède furent imaginées sur le même modèle : une femme drapée à l'ancienne, dans une situation dynamique, entourée de l'ensemble des attributs reliés à la discipline illustrée. Pour Erato (muse de la poésie amoureuse), la flèche d'Eros sert de plume et le personnage de l'Amour lui-même souffle les vers à l'oreille de la muse. Pour Polymnie (muse de l'Éloquence), la tribune, le diadème et le sceptre agissent comme signes de son pouvoir seigneurial sur la multitude, pouvoir conquis par les qualités de son discours. Les femmes de Vien (illustration 2), moins austères des points de vue formel et argumentaire, n'en demeurent pas moins précurseurs des Muses de Meynier, qui actualise un modèle standard, celui de la figure féminine impersonnelle. Réceptacle visuel de l'agrément, du confort, voire parfois de l'érotisme, la figure féminine s'avère le motif type de la décoration intérieure, quel qu'en soit l'habillage conceptuel qui, lui, est tributaire de l'esprit du temps : les caprices de l'amour chez la maîtresse de Louis XV, les disciplines humanistes agrémentées d'un retour de l'abondance chez les parvenus du Directoire comme Lannoy (illustration 3, le Salon de la richesse) et Boyer-Fonfrède. Dans le même esprit, Ingres - élève de David à la longue carrière - emporta ce modèle au cœur du XIX ${ }^{\mathrm{e}}$ siècle puisqu'il projetait de peindre La naissance de la dernière Muse (1856, aquarelle marouflée sur cuivre, 25,7 x 53,2 $\mathrm{cm}$, Paris, musée du Louvre) pour décorer la maquette d'un temple de Terpsichore, maquette que le prince Napoléon (frère de l'Empereur) avait commandée à l'architecte Hittorff en 1855, pour l'offrir à la comédienne Rachel, dont il était épris ${ }^{14}$.

Ces panneaux décoratifs du Directoire s'inscrivent aussi dans une autre tradition picturale, celle du grand nu féminin tiré de la fable antique, tel qu'il avait été inauguré par Botticelli dans les deux panneaux des Offices : La naissance de Vénus et Le Printemps, mais surtout, dans un esprit peut-être un peu moins décoratif, par Titien dans ses multiples versions de Vénus et Danaé ${ }^{15}$. De Jacques Blanchard à François Boucher, le nu féminin devait s'implanter dans la tradition française jusqu'aux propositions remarquables d'Anne-Louis Girodet : Danaé (musée de Leipzig, illustration 4) et de François Gérard : Flore (musée de Grenoble, illustration 5) peu avant $1800{ }^{16}$. Le premier 
tableau fut entrepris à la demande de Percier et Fontaine, qui réalisaient la décoration intérieure de l'hôtel particulier de Charles Gaudin situé rue du Mont-Blanc (autrefois et depuis rue de la Chaussée d'Antin). Quant au second, je crois qu'il faut le considérer comme un pendant éventuel du premier : les dimensions sont proches ${ }^{17}$ et les thèmes étrangement ressemblants, d'autant que Girodet fit le choix de remplacer la pluie d'or d'Ovide par des bijoux et des fleurs parant la belle. Sans compter que les quatre artistes se retrouvèrent impliqués dans le même genre de décoration trois ans plus tard à la Malmaison de Joséphine, qui recourut aux deux décorateurs de Gaudin, après les avoir découverts chez lui, du temps où ils étaient voisins ${ }^{18}$. Mais nous reviendrons sur les deux Ossian conçus pour la demeure de la femme du Premier Consul.

Flore et Danaé (que ce soient des pendants, ou pas) s'inscrivent toutes deux dans le domaine du marché privé. En ce sens, elles se rattachent à la production artistique de la Révolution, qui aurait pu échapper au prisme politique ou à celui de l'actualité, si perceptibles dans la peinture contemporaine de Salon ou de concours. Cela conduit à envisager l'éventualité d'une iconographie apolitique sous la Révolution, dont la particularité serait de s'être exprimée dans le domaine de la décoration intérieure, qui autorisait le traitement, en grand, des amours des Dieux ou autres épisodes anacréontiques : ici Zéphyr et Flore, là Zeus et Danaé. En fait, il me semble qu'il faut aborder ces peintures décoratives, surtout dans le cas de Girodet et Gérard - garants du maintien de la grande peinture sous la Révolution - comme les seuls dépositaires de la tradition picturale mythologique. Durant cette période, où les lieux officiels d'exposition et de commande étaient accaparés par l'actualité, ces panneaux, héritiers d'une tradition iconographique ambitieuse, donnèrent une forme de noblesse à ces salons, enclaves de légèreté fort prisées de la société directoriale. D'ailleurs, la seule incursion de Gérard dans le sens d'une réconciliation d'un sujet fabuleux et d'une exposition publique a fini de le convaincre de se tourner exclusivement vers le marché privé, où il pouvait déployer un talent essentiellement gracieux. En effet, Psyché et l'Amour (Paris, musée du Louvre), conçu sans commanditaire et exposé au Salon de 1798, s'il connut un succès d'initiés (les critiques et les artistes), n'en resta pas moins sans acheteur. Toutefois, avant 1801, Pierre Fontaine, architecte, et Joachim Le Breton, historiographe de l'art contemporain, s'associèrent pour l'acquérir ${ }^{19}$. Il n'est pas surprenant d'ailleurs que ces deux connaisseurs aient été sensibles à la qualité de cette œuvre. Gérard, quant à lui, s'installa dans le rôle de portraitiste, ce qui lui permit d'éviter l'actualité politique, faute de pouvoir la contourner en présentant aux Salons des peintures mythologiques.

À la suite de la Révolution, les propositions stylistiques de Gérard et Girodet, qui avaient connu une fortune limitée par la destination privée de ces peintures, furent récupérées et systématiquement exposées. Prudhon (Psyché enlevée par les Zéphyrs, 1808, Louvre), David (Sapho, Phaon et l'Amour, 1809, Saint-Petersbourg, Ermitage), Guérin (Aurore et Céphale, 1810, Louvre, illustration 6, et Iris et Morphée, 1811, Saint-Petersbourg, Ermitage), David (L'amour et Psyché, 1817, Cleveland Museum of Art), Delorme (Céphale et Aurore, 1822, Sens), et d'autres s'approprièrent ces personnages de la fable à l'érotisme et la nudité glacés, qui firent l'enchantement des collectionneurs d'Empire comme Sommariva et Youssoupoff ${ }^{20}$. Aussi, les panneaux décoratifs du Directoire servirent de relais dans la transmission de la tradition mythologique entre l'Ancien Régime et l'Empire, car l'exposition publique ne leur était pas propice. 
11 En marge de la production d'auteur, qui intéresse plus particulièrement la création d'œuvres symboliques, il en est une autre moins exceptionnelle, mais tout aussi intéressante parce qu'elle relaie le sens des peintures historiques : la production de panneaux peints, dont les motifs sont des formules appauvries ou répétitives d'un standard fourni par le décorateur. Louis Lafitte, bien que peintre d'histoire par ailleurs, servit les ensembles décoratifs des architectes Percier et Fontaine : en 1800, il peignit les huit panneaux destinés à décorer la salle à manger de la Malmaison. Tous furent réalisés d'après des dessins de Charles Percier. Six représentaient des danseuses et des musiciennes de style pompeïen et les deux derniers étaient ornés d'un trépied surmonté d'un vase antique. De nouveau, la figure féminine occupait la plupart des cimaises de cette salle décorée sous le signe de la fête (illustration 7). Ces motifs faisaient d'ailleurs remarquablement écho aux muses de la frise Talma restée rue de la Victoire. Parallèlement, la même équipe de décorateurs offrit à Girodet l'opportunité de réaliser quatre panneaux des Saisons, thème opportun pour le cabinet de platine de la Casa del Labrador (ou maison du laboureur, profession particulièrement tributaire du cours du temps) à Aranjuez ${ }^{21}$. Ce fut vraisemblablement l'argent qui conditionna la nature de ces deux collaborations parfaitement contemporaines : le budget du roi d'Espagne, Charles IV, autorisait la participation d'un jeune maître en vue, tandis que le budget de la Joséphine de 1800 ne le permettait sans doute pas. Quoi qu'il en soit, Girodet s'offrit une variation importante, puisqu'il réunit des figures féminines (le printemps et l'automne) et des figures masculines (l'été et l'hiver), équilibre sexuel qui ne s'étendit toutefois pas à l'âge, puisque les femmes sont toutes deux jeunes, éthérées, tandis qu'Apollon, lui aussi dans la fleur de l'âge, a au moins deux générations de différence avec le vieillard de l'hiver ${ }^{22}$.

Symbolique des lieux

12 Ces multiples projets décoratifs révèlent donc le dynamisme du marché privé sous le Directoire et la constance du goût des trente dernières années du XVIII ${ }^{\mathrm{e}}$ siècle, malgré la Révolution. Ceci contredit certainement l'idée un peu schématique de cette période prétendument suspensive pour le marché privé de l'art contemporain. En revanche, si la production et le goût se sont plus ou moins bien maintenus après 1789 et après 1794 , la question de la destination de ces tableaux aux mythologies ou allégories érotiques (Danaé, Psyché, amours...), ou tout simplement légères (jeux d'enfants et autres saisons) impliquent une définition affinée de leurs lieux d'exposition ${ }^{23}$. La plupart des panneaux évoqués ici n'ont jamais été exposés au Salon, parce qu'ils furent conçus pour des espaces privés. Toutefois, comme on le comprend bien à la lecture des mémorialistes de l'époque et des publications de décoration intérieure ${ }^{24}$, l'intimité de ces lieux soigneusement ornés était feinte, puisqu'ils se transformaient occasionnellement ou chroniquement en salles de réception, où se percevaient alliances et réseaux d'influence du propriétaire ou de la maîtresse des lieux. Comme cela existait sous l'Ancien Régime notamment grâce à la coutume du " petit lever ».

Dans ce jeu de l'intimité feinte, Juliette Récamier fut fort habile, car elle proposait systématiquement aux invités nouvellement venus dans son hôtel particulier de la rue du Mont-Blanc (jadis propriété du ministre Necker) de visiter sa chambre décorée sous le signe de Psyché par Berthault et Percier (le premier étant l'élève du second). Ses invitations, racontées par les voyageurs ${ }^{25}$, révèlent bien la part faite à la mise en scène dans l'introduction d'autrui à ses richesses et à son luxe. 

visuel devait révéler une part personnelle de l'hôte, comme sa richesse et son goût, mais aussi ses liens avec la société, qui composait sa cour (les invités) et qui œuvrait à l'extérieur, sur la place publique. Les ensembles décoratifs de ces salles de réception racontaient donc les relations de ces nouvelles élites avec la sphère publique. Ils définissaient visuellement un espace de co-présence, de visibilité et d'influence. Par conséquent, les choix iconographiques des propriétaires et des artistes furent souvent conditionnés par le fait que ces pièces étaient des lieux où devaient naître les cercles, voire les réseaux politiques.

15 Sous le Directoire, on observe trois types de décoration qu'il faut distinguer selon des catégories de lieux : professionnel, public et privé. Percier et Fontaine, impliqués dans les projets d'ameublement et de décoration des parvenus de la Chaussée d'Antin, conçurent aussi la décoration de l'atelier d'Isabey ${ }^{26}$. Grâce au tableau de Louis-Léopold Boilly et aux planches du recueil de décoration de Percier et Fontaine, on connaît bien cet ensemble décoratif qui illustrait la double fonction du lieu : atelier et chambre à coucher. Sur les quatre allégories des Beaux-Arts qui décoraient la pièce (illustration 8 , planche $\mathrm{V}$ du recueil de 1812), le tableau de Boilly montre bien, derrière la corporation c'est-à-dire les artistes, celles de la peinture et de la sculpture qui se trouvaient de chaque côté de la tête de Minerve, alors qu'une frise de portraits de peintres célèbres se déployait sur la parcelle la plus haute du mur. Les deux allégories de la gravure et de l'architecture se trouvaient sur l'autre mur, tandis que le plafond accueillait, en pendants, Apollon, symbole du jour (côté fenêtre), et Diane, symbole de la nuit (côté lit) 27. Aussi, le programme décoratif de cet atelier s'articulait littéralement autour de la fonction de la pièce, à tel point qu'il s'apparentait à un intitulé visuel (ou rébus) du lieu.

D'autre part, l'espace imaginé par Boilly (à la demande d'Isabey, vraisemblablement) nous oblige à souligner les correspondances entre cette pièce virtuelle - ce n'est qu'un projet de décorateurs au moment où Boilly peint le tableau - et cet atelier artificiel, sans élèves et sans objets adéquats, tels les chevalets, les modèles, les pinceaux... En fait, cette réunion fictive d'artistes - car il est peu probable que Boilly ait réussi à rassembler tous ces hommes dans une même pièce et au même moment - conduit à reclasser le lieu : d'atelier privé, il devient club corporatiste, dont les panneaux décoratifs correspondent à la liste des membres (les portraits) et à des enseignes (les quatre allégories des Beaux-Arts).

Dans le domaine de la décoration, la connivence de la fonction et du décor apparaît être une nécessité, surtout dans les cas (rarissimes sous le Directoire) de la décoration publique ${ }^{28}$. Aussi, lorsque le Directoire décida de convertir les appartements d'été d'Anne d'Autriche, remarquablement décorés par Romanelli et Anguier au XVII siècle, en musée des antiques, l'architecte Raimond (affecté au Louvre) commanda des tableaux à Philippe Hennequin (L'Hercule français, illustration 9), Guillaume GuillonLéthière (La Victoire et le Génie des arts), Pierre Peyron (L'étude éclaire et instruit l'émulation) et Pierre Guérin (Médaillon avec deux amours : génies de l'immortalité), pour définir visuellement la nouvelle fonction de ces salles. Il est alors question des conquêtes de l'armée française (Hennequin), de la victoire militaire octroyant au pays vainqueur le génie des arts (Léthière) et des possibilités offertes aux artistes fréquentant assidûment les chefs-d'œuvre éternels (Peyron et Guérin). Le programme réussit à justifier l'étonnant butin de guerre et à vanter les mérites de cette nouvelle «implantation", à la fois française et publique, des antiques. Dans cet ensemble 
décoratif (1798-1800), on retrouve une rhétorique présente dans les tableaux de concours de la Révolution, comme La France triomphante encourageant les sciences et les arts au milieu de la guerre de Charles Meynier (concours de l'an II, esquisse, Boulogne, Bibliothèque Marmottan) ou La liberté parcourant le monde de Jacques Réattu (1798, Arles, musée Réattu, pendant d'un tableau de concours de l'an II : Le triomphe de la civilisation, New York, collection particulière). Cette rhétorique se nourrissait de fierté nationale et d'utilité publique, habiles justifications de moyens contestables, comme l'emploi de la force et la saisie d'œuvres d'art.

De même qu'il est une différence catégorielle entre le salon privé et l'institution publique (le musée), il est une différence formelle entre leurs décors respectifs, car les artistes engagés pour affecter visuellement les anciens appartements d'Anne d'Autriche ne recoururent pas à la figure féminine impersonnelle, motif phare de la décoration privée. Effectivement, s'il y eut des commanditaires masculins et féminins, tels Charles Gaudin et Juliette Récamier, qui firent tous deux décorer leurs hôtels de la rue du MontBlanc, ils furent conçus, indépendamment du sexe du client, sous le même signe de l'univers féminin : Danaé pour Gaudin, Psyché pour Juliette, car, semble-t-il, ce motif caractérisait efficacement l'espace domestique, privé et plaisant.

Les correspondances entre la place de la femme dans la société d'après 1794 et les choix de ces belles captives (Psyché et Danaé) comme moteurs de la décoration domestique invitent à une interprétation du réseau d'influence de ces «Merveilleuses» et de leur pouvoir indirect sur la sphère publique.

Dans la fable antique, Danaé et Psyché éprouvèrent deux expériences communes : le ravissement et la captivité. Danaé fut enfermée dans une tour d'airain par son père, qui redoutait sa descendance, car l'oracle lui avait prédit qu'il serait tué par son petit-fils. Et, Persée, issu de l'union de Zeus métamorphosé en pluie d'or et de Danaé, réalisa effectivement la prédiction ${ }^{29}$. Quant à Psyché, elle fut enlevée par l'Amour, qui l'installa dans un palais magnifique, tout en lui interdisant de chercher à connaitre son identité et à voir les traits de son visage ${ }^{30}$. La jeune femme ne put résister à sa curiosité (excitée par ses sœurs envieuses), perdit son amant et dut, pour le reconquérir et devenir son épouse éternelle, subir toute une série d'épreuves imposées par Vénus, dont la jalousie et les colères avaient toujours été dirigées contre de jeunes et belles mortelles. Dans le cas où la Flore de Gérard appartint aussi à la production décorative du Directoire, il faut la joindre à cette étude consacrée à l'iconographie domestique de la société post-jacobine. Flore fut elle aussi enlevée par Zéphyr, qui l'épousa en justes noces et récompensa sa docilité en lui offrant le royaume des fleurs ${ }^{31}$. Elle sut d'ailleurs mettre à profit ce règne, puisqu'elle offrit à Junon une fleur dont le simple attouchement permit à la déesse de concevoir un enfant mâle, Mars, sans avoir eu de rapport avec Zeus.

21 L'érudition littéraire d'artistes comme Girodet et Gérard ${ }^{32}$ permet de supposer que la plupart des auteurs de ces ensembles décoratifs connaissaient par le menu ces fables amoureuses, dont ils pouvaient tirer parti dans la mise en scène d'un règne privé, en marge de l'arène politique exclusivement réservée aux hommes. Tacitement, ils établirent une correspondance entre les salons privés des hôtels particuliers de Mesdames Tallien, Récamier, Bonaparte... et les cages dorées de ces belles mortelles : Psyché, Danaé et Flore, enlevées à leur monde d'origine pour être installées dans un monde clos, où leur rapport à l'extérieur était entièrement médiatisé par la sexualité : l'union à Zeus pour Danaé ; l'union à l'Amour pour Psyché et l'union à Zéphyr pour 
Flore. Ces royaumes clos de la fable antique faisaient écho aux territoires privés, où se déployait le pouvoir érotique de ces «Merveilleuses», qui agissaient ainsi sur la société invitée, et par voie de conséquence, sur la sphère publique.

Dans ces décors, le pouvoir érotique pouvait pallier l'éviction des femmes de la place publique. Car, en effet, depuis l'automne 1793, la fermeture des clubs politiques féminins, et, indifféremment, les exécutions de Marie-Antoinette et Olympe de Gouges s'étaient succédé dans un rejet commun et apolitique d'une éventuelle place des femmes dans le monde politique ${ }^{33}$. Aussi, Mesdames Tallien, Récamier, Bonaparte et autres séductrices s'approprièrent ces règnes privés, où la conquête érotique, troquée contre un pouvoir matériel (la pluie d'or ou le palais de l'Amour), permettait une entrée indirecte dans la vie publique. Le Directeur Barras et Madame Tallien, surnommée non sans hasard la Reine du Directoire après avoir été Notre-Dame-de-Thermidor, apparaissent comme le couple emblématique de cette dynamique directoriale de la différence des sexes, qui contournait la frontière étanche, mais artificielle, que les Jacobins avaient tenté d'ériger entre les hommes et les femmes dans le domaine politique.

Le salon, lieu de la réversibilité des genres

23 Les ensembles décoratifs liés au pouvoir indirect des femmes - majoritaires sous le Directoire - furent bientôt remplacés par des panneaux jouant toujours sur l'hybridité des lieux, à la fois privés et publics, et sur l'économie sexuelle des responsabilités politiques, mais sur un mode inversé. En fait, ils furent réalisés dans un registre intimiste, chargé de véhiculer une image romantique du héros militaire. Les Bonaparte furent les premiers à interpeller - volontairement ou pas, d'ailleurs - l'imagination des artistes dans le sens d'une image intime.

24 Après le 18 brumaire, Joséphine, consciente de l'importance croissante de son mari dans le destin militaire et politique de la France, passa commande à Percier et Fontaine pour son petit château de la Malmaison. Ceux-ci avaient déjà sollicité Girodet pour un panneau de la décoration Gaudin et lui renouvelèrent leur confiance. Cependant, le peintre, très inventif dans la mise en image d'idées sophistiquées, mais souvent mal compris et décalé par rapport aux intentions de ses commanditaires, imagina de traiter d'Hercule tuant Cacus (illustration 10).

Dès 1792, le personnage d'Hercule avait alimenté l'iconographie jacobine d'un peuple colossal capable de confronter le «monstre royal ou le géant monarchique », aussi on imagine mal comment Bonaparte (même celui de 1799) et l'Hercule populaire et démocratique conçu par Jacques-Louis David en 1794 auraient pu fusionner ${ }^{34}$. De plus, le personnage de Cacus, que Hercule est en train de terrasser dans l'esquisse peinte par Girodet, est un monstre à trois têtes. Soit Cacus représentait l'alliance des couronnes étrangères contre la France (les Anglais, les Russes, les Autrichiens et les Prussiens) ; soit l'image traitait métaphoriquement du danger intérieur, c'est-à-dire des conséquences de l'attentat de la rue Saint-Nicaise survenu le 24 décembre 1800. Bonaparte profita de cette tentative d'assassinat pour anéantir les derniers républicains, qu'il fit arrêter et exécuter hâtivement, alors que l'enquête tendait à montrer qu'ils n'en étaient pas responsables.

D'après le premier biographe de Girodet, l'hypothèse de la représentation allégorique de l'attentat de la rue Saint-Nicaise est la plus probable ${ }^{35}$. La proximité intellectuelle de Girodet avec le groupe de littéraires animant la revue Veillée des Muses ${ }^{36}$, dont le comité de rédaction était composé entre autres des écrivains Arnault, Deguerle, Lebrun- 
Pindare et des peintres David, Régnault, Isabey, abonde aussi dans le sens d'une illustration de l'attentat de la rue Saint-Nicaise. Effectivement, au moment même où Girodet esquissait son tableau, Crouzet écrivait une Ode sur l'attentat du 3 nivôse, qu'il lut à l'Institut le 3 pluviôse an IX (23 janvier 1801) ${ }^{37}$. Deguerle rendit compte de cette lecture dans un numéro de la Veillée des Muses ${ }^{38}$. Il souligna la qualité littéraire de ce texte - qu'il trouvait empreint d'un sublime à la Longin - et la clairvoyance politique de Crouzet, qui fit volontairement de Bonaparte un nouvel Hannibal, tout en attribuant l'organisation de cet attentat au gouvernement britannique. Girodet entendit sans doute l'appel de Deguerle, qui, évoquant les applaudissements suscités par cette lecture, ajouta que « son succès doit encourager les Muses timides qu'effrayait le bruit des armes - la paix, conquise par la victoire, va rendre à leur antique honneur, les sciences et les arts - les grands poëtes ont été dans tous les âges contemporains des héros ; et toutes les espèces de gloire doivent illustrer le siècle de Bonaparte ${ }^{39}$.

Toutefois, comment Girodet a-t-il pu penser que Joséphine souhaiterait cohabiter avec une telle peinture, parfaitement opposée à ses goûts coquets et étrangère à la fonction décorative d'un tableau de salon privé ${ }^{40}$ ? Dans une méprise complète de la destination du tableau, Girodet avait imaginé une peinture pour un lieu public : édifiante, allégorique, efficace voire simpliste. Hennequin avait lui aussi prolongé la fortune d'Hercule sous le Directoire, cependant, il l'avait intégré plus habilement que Girodet, car, non seulement le peintre lyonnais avait eu l'intelligence de proposer un panneau herculéen pour un édifice public (le plafond du nouveau musée des antiques), mais de plus, il en avait allégé la forme davidienne notamment pour le coloris, aérien dans le panneau du Louvre (illustration 9).

Quant à l'esquisse de Girodet, avant même d'être soumise à l'approbation de Joséphine, les architectes Percier et Fontaine la refusèrent. Cette maladresse donna au peintre l'occasion de peindre un tableau encore plus original, nouveau en France, et en adéquation avec le salon privé d'un couple princier, en l'espèce avec l'espace de représentation d'un souverain simulant l'intimité. Girodet conçut Ossian accueillant les Héros morts pendant la guerre de Liberté (illustration 11, 1801, musée national du château de Malmaison) qui devait faire pendant au Songe d'Ossian de François Gérard dans le Salon doré de la Malmaison ${ }^{41}$.

29 Bonaparte était un lecteur fervent de l'Ossian de Macpherson (faux recueil de textes gaéliques anciens narrant les exploits guerriers et les amours d'une tribu calédonienne du IIIe siècle après Jésus-Christ). Le Premier Consul aurait même déclaré : « Alexandre a choisi Homère pour son poète, Auguste a choisi Virgile, pour moi je n'ai eu qu'Ossian, les autres étaient pris !» ${ }^{42}$. Cette appropriation semble avoir été assez audacieuse puisque Ossian ne connut pas un succès immédiat en France, même si Letourneur l'avait traduit dès 1777. Les propos d'Antoine-Vincent Arnault, auteur d'une pièce intitulée Ossian, fils d'Oscar ${ }^{43}$, confirment cette impression, car, en 1796 , sa pièce avait connu une réception mitigée, dont il rendit compte dans sa préface : « les poëmes d'Ossian ne se trouvaient pas dans toutes les bibliothèques; son style, employé pour la première fois au théâtre, a dû étonner la multitude. Ce qui pour les gens instruits n'était que nouveau, pour le commun des spectateurs était étrange $"{ }^{44}$.

Aussi, Bonaparte choisit cet «Homère du Nord", car il lui permettait de se projeter dans une épopée romanesque encore inédite, contrairement à l'histoire et la mythologie greco-romaines, que les souverains anciens et modernes avaient déjà surexploitées. En même temps, le côté fantastique de cette littérature guerrière, truffée 
d'événements extraordinaires (apparitions, métamorphoses, géants, chimères...) ne pouvait se révéler que dans un contexte peu protocolaire. D'ailleurs, Bonaparte - et Napoléon encore moins - n'autorisa jamais l'allégorie ossianique dans les galeries de peintures constituées de ses hauts faits militaires. Le salon de Joséphine permettait la représentation métaphorique d'un guerrier invincible, mais aussi mélancolique, amoureux, entouré de personnages fantomatiques. Il est d'ailleurs assez intéressant d'observer que, à en croire l'auteur de l'Histoire naturelle de la femme (1803), JacquesLouis Moreau de la Sarthe, ces qualités étaient considérées comme l'apanage de ce sexe ${ }^{45}$. Aussi, l'image intime du héros militaire le révélait sensible aux mondes occultes et surnaturels, et par conséquent en partie féminisé.

31 En revanche, pour son domicile parisien officiel, le Palais des Tuileries, le Premier Consul circonscrit scrupuleusement son image à celle du général victorieux : en juillet 1800 , il demandait à son frère Lucien, ministre de l'Intérieur, de « choisir les meilleurs peintres pour faire peindre les batailles de Rivoli, Marengo, Messkirch, Pyramides, Aboukir et Mont-Tabor $»{ }^{46}$.

32 Assurément, l'ossianisme de Bonaparte, révélé dans le salon de Joséphine par les deux peintures de Gérard et Girodet - comme plus tard pour la chambre de Napoléon dans le palais du Quirinal, où Ingres réalisa un plafond peint illustrant à nouveau le Songe d'Ossian (1812, Montauban, musée Ingres) - devait fonctionner comme la part intime à rendre publique, via le cercle des invités. Ingres fut décidément l'artiste qui porta le plus avant dans le XIXe siècle la rhétorique mise au point par la première génération d'élèves de David, comme l'avait déjà montré sa Naissance de la dernière Muse pour Rachel.

33 Les panneaux décoratifs conçus sous le Directoire s'inscrivent dans la tradition picturale moderne, car ils s'approprièrent le vocabulaire formel fondé sur la figure féminine. Toutefois, pendant la Révolution française, cette production artistique privée, ancrée dans des conventions de plusieurs siècles, se révéla aussi en correspondance avec l'évolution politique exceptionnelle, notamment quant à la répartition des rôles entre les hommes et les femmes. Aussi, l'étude de cet aspect privé renseigne autant sur les ruptures politiques que sur les continuités domestiques (et vice versa) propres à la Révolution. De même, l'hybridité de ces lieux de réception, que furent les salons privés, permit sans doute un rééquilibrage des tâches strictes, que la société jacobine avait léguées aux citoyens et aux citoyennes. Pour échapper à ce clivage artificiel : aux hommes la sphère politique, aux femmes la sphère privée, les «Incroyables» et les «Merveilleuses» inventèrent des lieux en retrait, où ils transgressaient ce séparatisme rigide.

\section{NOTES}

1.Pour faciliter la compréhension de cet article, je tiens à préciser que j'emploierai le mot « Salon » (avec une majuscule) seulement pour indiquer l'exposition publique 
annuelle du Louvre et « salon privé » (avec une minuscule) pour désigner la pièce d'un appartement ou d'un hôtel particulier.

2.Philippe Bordes et Régis MicheL, Aux armes et aux arts! Les arts de la Révolution 1789-1799, Paris, 1988; La Révolution française et l'Europe, cat. d'exp., 1989, Paris, Grand Palais. Édouard Pommier, L'art de la Liberté Doctrines et débats de la Révolution française, Paris, 1991; Thomas CRow, L'atelier de David Émulation et Révolution, Paris, 1997 (Londres, 1995).

3.Gabriel-Julien Ouvrard (1770-1846) constitua sa fortune grâce au commerce colonial ; le marquis Bernard François de Chauvelin (1766-1832), ambassadeur à Londres en 1792 (à la même époque que Charles Percier), appartenait à une famille de grands commis d'État et Martin-Michel-Charles Gaudin (1756-1841) fut ministre des Finances sous l'Empire puis gouverneur de la Banque de France sous la Restauration.

4.Pierre FontAINE, Journal 1799-1853, Paris, 1983, vol. 2, p. 1133.

5.R. La MAŻ̀RE, « Les hôtels de la Chaussée d'Antin », L'illustration, 18 décembre 1843; Gustave BORD et Louis BIGARD, La maison du dix-huit brumaire, Paris, 1930; Jocelyne van DePUTTE, Vie et histoire du IXe arrondissement, Paris, 1986; Jean-Marie PÉRousE de Montclos, dir., Le guide du patrimoine, Paris, Paris, 1994. Maryse GolDEMBERG, Le guide du promeneur 9e arrondissement, Paris, 1997.

6.Louis-Sébastien Mercier consacrait un de ses premiers chapitres du Tableau de Paris à la rue Vivienne qu'il qualifiait ainsi : «Il y a plus d'argent dans cette seule rue que dans tout le reste de la ville ; c'est la poche de la capitale » (édition de 1990, Paris, Robert Laffont, collection «Bouquins », p. 291) et plus loin, dans une langue explicite, il ajoutait : « ce qui compose l'agiotage, et toute cette race ennemie de la sainte agriculture, se loge aux environs de cette rue pour être plus à porter des autels de Plutus. Les catins y sont plus financières que dans tout autre quartier, et distinguent un suppôt de la bourse à ne pas s'y tromper » (ibid.).

7.En effet, la Chaussée d'Antin avait commencé à susciter l'intérêt des investisseurs immobiliers et de personnages moteurs du goût et de la mode parisienne (les riches fermiers généraux, les banquiers, les nobles de robe et leurs maîtresses souvent comédiennes ou danseuses comme Mademoiselle Dervieux et Julie Talma) à la suite de l'expropriation de petits artisans et de petits commerçants provoquée sous la Régence par l'installation de la Cour au Louvre. En conséquence, le quartier palatial dut être réorganisé et la limite de l'enceinte de Paris reculée jusqu'à la Ville l'Évêque, la Grange Batelière et la rue des Porcherons, où s'établirent ces nouveaux riches (sources citées à la note 5).

8.Joséphine et Napoléon Bonaparte rachetèrent à Julie Carreau leur maison de la rue Chantereine (future rue de la Victoire) le 31 mars 1798, mais ils en étaient locataires depuis trois ans. Le général Moreau racheta l'hôtel Lakanal (bâti par Henry en 1795 et sis rue du Mont-Blanc) le 25 frimaire an VIII (16 décembre 1799); il le revendit le 27 thermidor an IX (15 août 1801) alors qu'il en avait acquis un autre rue d'Anjou cinq mois auparavant, le 17 ventôse an IX (8 mars 1801). Hortense et Louis Bonaparte achetèrent en 1802 un hôtel sis rue Joubert qui avait été édifié en 1777 pour Mademoiselle Dervieux (danseuse, maîtresse du comte d'Artois puis épouse de l'architecte Bélanger), hôtel que la comédienne Mademoiselle Lange avait aussi habité en 1798. Le cardinal Fesch emménagea aussi dans ce quartier en $1802:$ il acquit l'hôtel (édifié en 1765) de l'ancien président du Parlement Hocquard de Montfermeil ; quant à Murat, il racheta l'hôtel de Madame de Thélusson (édifié par Ledoux en 1782) en 1803 (sources citées à la note 5). 
9.Je fais ici référence au fameux studiolo de Leonello d'Este (Ferrare, palais du Belfiore) dont la décoration, imaginée par Guarino Veronese et réalisée par des peintres proches de Cosmè Tura, tenait à la représentation de neuf muses (Le muse e il Principe, cat. d'exp., Milan, Musée Poldi Pezzoli, 1991). De même, le salon des Saisons de Pierre-Paul Prudhon est bien connu grâce aux études de Sylvain Laveissière (chapitre VI " Décorations » du cat. d'exp. Prud'hon ou le rêve du bonheur, 1997, Paris, Grand Palais, pp. 137-155) et de Helen WESTON (chapitre 7 « Working for a bourgeois republic : Prud'hon, patronage and the distribution of wealth under the Directoire and Consulate ", dans William VAUGHAN et Andrew HEMINGWAY (dir.), Art in a Bourgeois Society 1790-1850, Cambridge, 1998, pp. 154-177).

10.Cette peinture à fresque datée du premier siècle avant Jésus-Christ fut découverte sous le pontificat de Clément VIII, près de la Basilique Sainte-Marie-Majeure de Rome, dans les anciens jardins de Mécène. Ce fut un modèle connu et souvent commenté, d'autant que les exemples de peinture antique étaient rares : Poussin la copia ; Carloni la grava en couleurs sur cuivre et elle fut reproduite dans l'ouvrage de Bernard de Montfaucon, L'Antiquité expliquée et représentée en figures, Paris, 1719, tome III, 2e partie, chapitre IX, livre I, planche CXXIX, p. 220.

11.Gustave BORD et Louis BIGARD, La maison du 18 brumaire, op. cit., pp. 92-107.

12.En 1773, la comtesse du Barry refusa la série de peintures décoratives consacrées aux progrès de l'amour que Jean-Honoré Fragonard avait conçue pour son pavillon de Louveciennes, et remplaça le maître des polissonneries par le peintre "à l'antique ", Joseph-Marie Vien, qui traita du même sujet sur un mode néoclassique. Les tableaux de Fragonard sont à la Frick collection de New York, tandis que ceux de Vien sont partagés entre le musée du Louvre et la préfecture de Chambéry.

13.Ces panneaux seront publiés prochainement par Isabelle Meyer-Michallon, qui termine une thèse sur Charles Meynier (Université Paris-IV), tandis que Amy Freund consacrera un long chapitre de sa thèse sur le portrait sous la Révolution et l'Empire (Los Angeles, Université de Californie) à François-Bernard Boyer-Fonfrède, portraituré par Vincent (1801, Versailles, musée national du château) qui était déjà l'auteur d'un portrait de l'épouse et du fils Boyer-Fonfrède (1796, Paris, musée du Louvre).

14.Georges VIGNE, Ingres, Paris, 1995, p. 291, repr.

15.La Vénus endormie de Giorgione (v. 1508, Dresde, Gemäldegalerie) est bien sûr le tableau fondateur de cette série féminine. Suivent de nombreux tableaux de Titien : Flore (1515, Florence, musée des Offices) ; La Vénus d'Urbino (1538, Florence, musée des Offices) ; Danaé (1545, Naples, Capodimonte) ; Danaé (1553, Madrid, Prado) ; Danaé (1554, Saint-Petersbourg, Ermitage) et Danaé (1554, Vienne, Kunsthistorisches Museum). 16.Malgré la date erronée de 1798 (date avancée par le premier biographe de Girodet : Pierre-Alexandre coupiN, « Notice historique... » dans CEuvres posthumes de GirodetTrioson, Paris, 1829, vol. I, p. LVJ), la première Danaé fut assurément réalisée en 1797 : le tableau est daté de l'an VI et sa lithographie, réalisée par Aubry-Lecomte sous la direction du maître en 1824, porte la lettre suivante : Girodet-Trioson ping.t 1797 et dir.t. Pour la Flore, on sait, grâce à la lettre d'une gravure de Charles-Simon Pradier d'après un tableau perdu (Paris, Bibliothèque nationale de France, cabinet des estampes, Gérard SNR), que la première version peinte fut réalisée en 1799. En revanche, la peinture de Grenoble daterait de 1802.

17.Danaé: 170 x $89 \mathrm{~cm}$ et Flore : 170 x $100 \mathrm{~cm}$. D'autre part, la toile de Girodet pourrait avoir été coupée sur sa largeur, car l'une des trois figures du motif en bas relief, qui se trouve en bas à gauche, est visible seulement à mi corps tandis que la lithographie 
d'Aubry-Lecomte confirme que la peinture originale était plus large. Après 1824 (date de la lithographie), la toile aurait été réduite pour convenir à un nouvel emplacement. 18. FONTAINE, op. cit. à la note 4 .

19.T. C. BRUUn NEERGAARD, Sur la situation des Beaux-Arts en France..., Paris, 1801, p. 116; Lettres adressées au baron François Gérard..., Paris, 1886, vol. 1, « Notice biographique », p. 8.

20.Le Prudhon, le Guérin de 1810 et le David de 1817 faisaient partie des collections de Sommariva tandis que le David de 1809 et le Guérin de 1811 appartenaient à Youssoupoff. À l'exception de l'Amour et Psyché de David qui fut exposé à Bruxelles en 1817, tous ces tableaux ont été exposés au Salon de Paris, l'année même de leur réalisation.

21.Chantal GASTINEL-COURAL, « Le cabinet de platine de la Casa del Labrador à Aranjuez Documents inédits ", Bulletin de la société de l'histoire de l'art français, 1993-1994, pp. 181-205.

22.Girodet donnera une version similaire de ces Saisons à l'occasion de la décoration de la chambre de l'Impératrice pour le château de Compiègne, en 1814. (Jacqueline PRUVOST-AUZAS, « Les peintures de Girodet au Palais de Compiègne », Bulletin de la société de l'histoire de l'art français, 1969, pp. 98-99 et 101, repr.).

23.Les six amours peints par François Gérard pour le salon privé de Madame Tallien ont réapparu à la vente du général Rapp du 11 mars 1822, Paris, $n^{\circ} 11$ à 17.

24.Duchesse d'ABRANTÈs, Histoire des Salons de Paris, Paris, 1838, tome III, (commentaires sur l'hôtel Thélusson, l'hôtel de Richelieu, le salon de Barras et madame Tallien, la maison Seguin, rue d'Anjou, et l'hôtel Lannoy...); Ch. PERCIER et P. FONTAINE, Recueil de décoration intérieure, Paris, an IX (1801); J.-Ch. KRAFFT et N. RANSONNETTE, Plans, coupes et élévations des plus belles maisons et des hôtels construits à Paris et dans ses environs entre 1771 et 1802, Paris, 1802.

25.J. F. REICHARDT, Un hiver à Paris sous le Consulat 1802-1803, Paris, 1896, pp. 96-99 et Madame de CAZENove d'ARLENS, Deux mois à Paris sous le Consulat (février-avril 1803), Paris, 1903, p. 79.

26.Boilly 1761-1845. Un grand peintre français de la Révolution à la Restauration, cat. d'exp. rédigé par Annie SCOTTEZ-DE WAMBRECHIES et Sylvain LAVEISSIÈRE Lille, musée des BeauxArts, 1989, «L'atelier d'Isabey : un panthéon de l'amitié », pp. 50-79.

27.Les cinq premières planches du Recueil de décorations intérieures de Percier et Fontaine (Paris, 1812) instruisent parfaitement l'aménagement de cette pièce.

28.Il n'y eut pas beaucoup de décoration d'espaces publics parce que la conception et la réalisation de programmes décoratifs ambitieux nécessitent du temps, or la Révolution française ne connut pas la durée.

29.ovIDE, Les métamorphoses, livre IV, vers 610-612.

30.APULÉE, L'âne d'or ou Les Métamorphoses, livres V et VI.

31.ovide, Fastes, Livre V, vers 183-378.

32.La thèse de Sidonie LEMEUX-FRAITOT (Ut poeta pictor, Université Paris-I, direction d'Éric Darragon) a bien montré l'étendue de la culture littéraire de Girodet, de même que les nombreuses collaborations de ces artistes avec les éditeurs Didot pour les illustrations des textes de Jean de la Fontaine (Les amours de Psyché et Cupidon, 1797, planches d'après des dessins de Gérard), Virgile (1798, planches d'après des dessins de Gérard et Girodet), Horace (1799, planches d'après des dessins de Charles Percier) et Racine (1801, 
planches d'après des dessins de Gérard, Girodet, Chaudet, Taunay, Moitte, Peyron, Prudhon et Serangeli) confirment leur érudition.

33.Lynn Hunt, Le roman familial de la Révolution française, Paris, 1995, pp. 130-139.

34.Id. Politics, Culture and Class in the French Revolution, Berkeley, 1984, pp. 95-113 et

Antoine de BAECQUE, Le corps de l'histoire métaphores et politique (1770-1800), Paris, 1993, pp. 376-389.

35.P.-A. CoupIN, « Liste des principaux ouvrages de Girodet », dans CEuvres posthumes de Girodet-Trioson, op. cit. à la note 16, p. LXX.

36.Cette revue de critique littéraire et de création a paru entre 1797 et 1801 (douze volumes). Elle fut animée et rédigée par Lebrun-Pindare, Arnault, Laya, Deguerle, Dorat, Le Gouve et Vigée, qui se réunissaient tous les quinze jours dans l'hôtel Thélusson de la rue de Provence, pour travailler à chaque numéro d'une centaine de pages.

37.Pierre Crouzet (1753-1811), prêtre constitutionnel, fut l'auteur de quelques hymnes patriotiques.

38.Veillées des muses, $\mathrm{n}^{\circ} \mathrm{XI}$, nivôse an IX, pp. 206-216. D'autre part, les liens de J.N.M. Deguerle (1766-1824) et Girodet (1767-1824) devraient certainement faire l'objet d'une étude plus approfondie, car l'homme de lettres est aussi l'auteur d'un Stratonice et son peintre, ou Les deux portraits, conte qui n'en est pas un, Paris, an VIII (1801), dont les ressorts semblent directement empruntés aux circonstances particulières de la réalisation du Portrait de Mademoiselle Lange en Danaé (Girodet, 1799, Minneapolis Institute of Arts).

39.Ibid., p. 216.

40.La récente exposition d'Alain Pougetoux à la Malmaison (La collection de peintures de l'impératrice Joséphine, 2003, pp. 42-49) nous a montré que Joséphine préférait les genres mineurs et précieux, telle la peinture troubadour, à la peinture d'histoire.

41.Gérard a conçu quatre versions de son Ossian évoquant les fantômes au son de sa harpe. Seules deux sont encore connues : celle conservée au château de Rueil-Malmaison et celle de la Kunsthalle de Hambourg ; une troisième, jadis à Berlin, aurait été détruite par les bombardements de la ville en 1945. On ignore aussi laquelle de ces quatre peintures fit partie de la collection d'Eugène de Beauharnais.

42.Citation prise dans un article d'Hélène Toussaint, « Notes Liminaires », La légende d'Ossian illustrée par Girodet, cat. d'exp., Montargis, musée Girodet, 1989, p. 15.

43.Cette tragédie en cinq actes fut mise en musique par Méhul et créée au Théâtre de la République le 14 prairial an IV (3 juin 1796).

44.A.-V. ARnault, Euvres, Paris, 1824-1827, vol. III, préface à Ossian, fils d'Oscar, p. 227. 45.Voici ces mots rapportés par Lynn Hunt, (op. cit. à la note 34, 1995, p. 178) : « les femmes sont plus disposées que les hommes à croire aux esprits, et à avoir des apparitions ; elles se livrent d'autant plus aisément à toutes les pratiques superstitieuses que leurs préjugés sont plus nombreux ».

46.Napoléon BONAPARTE, Correspondance, Paris, 1861, vol. 6, p. 408, lettre n 4993 : « au citoyen Lucien Bonaparte, ministre de l'intérieur », 27 messidor an VIII (16 juillet 1800). 


\section{RÉSUMÉS}

Cet article étudie la place et la fonction de la décoration privée sous le Directoire. Les œuvres originales des peintres Girodet, Gérard, Prud'hon, Meynier et, moins célèbres, les panneaux de Lafitte inspirés des modèles de Percier et Fontaine en forment le corpus principal. Contrairement aux idées reçues sur la rupture de la Révolution française, cette production s'inscrivit dans la tradition décorative, au point que la filiation stylistique fut tout à fait linéaire dans le quartier de la Chaussée d'Antin entre 1770 et 1800 . La deuxième partie de cette étude porte, d'une part, sur les lieux d'exposition de ces panneaux : salons privés, qui fonctionnaient alors comme des lieux intermédiaires entre sphère privée et sphère publique; et, d'autre part, sur les constantes iconographiques de ces ensembles censés relayer l'influence des « Merveilleuses » après leur exclusion de l'arène politique.

In Search of an "incredible " and " marvellous" Iconography. The Decorative Panels during the Directoire Period. In this article, we are studying the place and the function of interior decoration during the Directoire period.The original works of painters such as Girodet, Gérard, Prud'hon, Meynier, and, to a lesser extend, Lafitte's panels inspired by Percier and Fontaine's models, constitute our main focus. Contrary to the usual perception concerning the total artistic break brought by the French Revolution, this production stays so close to the decorative tradition, that one can trace a clear line of descent in the decorative style in the Chaussée d'Antin District, between 1770 and 1800. The second part of this study is two pronged: First we examine the venues for the exhibitions of these panels, i.e. the private salons which occupied a sort of middle ground, between private and public space. Then we examine the constant iconographic elements present in these ensembles, which are supposed to replace the influence of the Merveilleuses, after they were excluded from the political arena.

INDEX

Mots-clés : Directoire, iconographie, ensemble décoratif, Chaussée d'Antin, mythologie, Genre, Gérard (François), Girodet (Anne-Louis), Récamier (Juliette), Bonaparte (Joséphine)

\section{AUTEUR}

\section{ANNE LAFONT}

Maître de conférences d'histoire de l'art moderne à l'Université de Marne-la-Vallée. Equipe de recherche: EA3350 Histoire comparée des pouvoirs. Bâtiment Bois de l'Etang, rue Galilée, 77420 Champs-sur-Marne cedex 